Revue européenne des sciences sociales

European Journal of Social Sciences

XLV-136 | 2007

Démocratie délibérative, démocratie débattante, démocratie participative

\title{
La décision par consensus apparent. Nature et propriétés
}

Philippe Urfalino

\section{CpenEdition}

Journals

Édition électronique

URL : http://journals.openedition.org/ress/86

DOI : $10.4000 /$ ress. 86

ISSN : 1663-4446

Éditeur

Librairie Droz

Édition imprimée

Date de publication : 1 février 2007

Pagination : 47-70

ISBN : 978-2-600-01114-3

ISSN : 0048-8046

Référence électronique

Philippe Urfalino, "La décision par consensus apparent. Nature et propriétés », Revue européenne des sciences sociales [En ligne], XLV-136 | 2007, mis en ligne le 01 février 2010, consulté le 19 avril 2019. URL : http://journals.openedition.org/ress/86 ; DOI : 10.4000/ress.86 


\section{Philippe URFALINO}

\section{LA DÉCISION \\ PAR CONSENSUS APPARENT Nature et propriétés ${ }^{1}$}

Les philosophes et les politologues dont les réflexions visent à promouvoir une démocratie délibérative privilégient un mode de décision collective le plus souvent dénommé «décision par consensus». Ils rejoignent sur ce point précis certains mouvements politiques alternatifs et protestataires (écologistes, féministes, mouvements de patients). Les uns et les autres estiment préférable que les décisions soient prises à l'issue d'une longue discussion permettant de dégager un consensus plutôt que par un vote, notamment à la majorité. Le consensus est préférable à la règle de majorité pour au moins deux raisons: le souci de parvenir à un consensus exige une écoute de tous les points de vue et permet plus facilement la participation de chacun à la discussion; la participation de tous à la délibération et l'exigence de consensus accroissent la qualité et la légitimité de la décision (Cohen [1989]). Il n'est pas rare que l'argumentation en faveur d'une démocratie délibérative fasse référence à des précédents historiques, et notamment à la prévalence dans presque toutes les sociétés «traditionnelles» de ce qu'on appelle en Afrique la palabre. Ainsi James Fishkin (1991) mentionne-t-il l'exemple des assemblées Quaker où la discussion continuait jusqu'à l'obtention d'un consensus, de telle manière qu'aucune décision ne pouvait être prise contre la volonté de l'un d'entre eux. De la même façon, Amartya Sen (2005), dans une réflexion sur les racines non occidentales de la démocratie, qui l'amène à en privilégier la dimension délibérative, illustre par la tradition de la palabre l'existence d'une culture politique du débat public et ouvert à tous, hors de l'Occident. Le lien entre démocratie et palabre, thème ancien, est réactivé via un extrait remarquable de l'autobiographie de Nelson Mandela. L'ancien leader de l'Afrique du Sud y explique que sa conception du commandement fut profondément influencée par le déroulement des réunions tribales de la société Thembu qu'il avait pu observer dans sa jeunesse:

«Tous ceux qui voulaient parler le faisaient. C'était la démocratie sous sa forme la plus pure. Il pouvait y avoir des différences hiérarchiques entre ceux qui parlaient, mais chacun était écouté, chef et sujet, guerrier et sorcier, boutiquier et agriculteur, propriétaire et ouvrier. Les gens parlaient sans être interrompus et les réunions duraient des heures. Le gouvernement avait pour fondement la liberté d'expression. ... Les réunions duraient jusqu'à ce qu'on soit arrivé à une sorte de

1 La rédaction de ce texte a grandement bénéficié des discussions que j’ai pu avoir avec Bernard Manin et Pasquale Pasquino. Par ailleurs, les commentaires de Catherine Alès, Alban Bouvier, Sébastien Dalgalarrondo, Boris Hauray, Philippe de Lara, Gabriel Nardacchione et Gudrun Urfalino m'ont permis d'améliorer une première version de ce texte. Je les en remercie tous très chaleureusement. 
consensus. Elles ne pouvaient se terminer qu'avec l'unanimité ou pas du tout. Cependant, l'unanimité pouvait consister à ne pas être d'accord et à attendre un moment plus propice pour proposer une solution. La démocratie signifiait qu'on devait écouter tous les hommes, et qu'on devait prendre une décision ensemble en tant que peuple. La règle de majorité était une notion étrangère. Une minorité ne devait pas être écrasée par une majorité » (Mandela [1995], pp. 29-31).

Le propos de cet article n'est pas de discuter la valeur démocratique de la décision par consensus ou d'examiner la possibilité de son application aux sociétés modernes. Mon propos est d'abord descriptif. L'objectif est de cerner la spécificité de ce mode de décision, notamment en essayant de le distinguer clairement du vote à la majorité et à l'unanimité. L'enjeu de cette description ne concerne pas seulement la réflexion sur la démocratie délibérative, il touche plus largement à la théorie des décisions collectives. Si un travail de description et de clarification conceptuelle me parait utile, c'est bien entendu parce qu'il me semble que ce travail n'a pas été intégralement accompli.

En effet, les réflexions sur la démocratie délibérative, aussi bien que les descriptions des ethnologues, souffrent dans la quasi-totalité des cas du même défaut. La séquence finale de ce qu'ils appellent la «décision par consensus » ou la «décision à l'unanimité » n'est évoquée que de manière allusive. Le lecteur est censé comprendre que, à la fin, un consensus permet de prendre la décision. Mais les auteurs ne décrivent pas la manière dont la décision est arrêtée, ils ne se sont pas donné les moyens de répondre à la question suivante: comment les participants se rendent-ils compte que, de fait, un consensus a été établi et donc que la décision collective est prise?

Dans une première partie, la présentation d'une série de descriptions disponibles dans la littérature permettra de dégager la spécificité de ce que je nommerai la décision par consensus apparent. Dans la seconde partie, j'étudierai les caractéristiques et les propriétés de ce mode d'arrêt de la décision collective qui, à l'inverse du vote, n'a pas pour préalable le dénombrement des opinions.

\section{UN PROBLÈME DE DESCRIPTION}

«Les Navahos n'ont pas la notion de gouvernement représentatif. Ils ont l'habitude de décider de toute question dans des rencontres de tous les individus concernés... Traditionnellement, ils ne prennent une décision qu'après en avoir discuté jusqu'à ce que l'unanimité soit réunie, ou jusqu'à ce que l'opposition trouve inutile de continuer à soutenir son point de vue ». Cette manière de prendre collectivement des décisions, décrite en 1946 par Clyde Kluckhon et Dorothea Leughton pour les Indiens Navahos ${ }^{2}$, semble bien avoir été le mode de décision le plus répandu dans l'histoire des sociétés humaines. L'ancienneté et la présence sur tous les continents de ce mode de décision dit tantôt «par consensus », tantôt «à l'unanimité », sont attestées par les travaux des ethnologues et des historiens. C'est le seul mode de décision mentionné pour les sociétés de chasseurs cueilleurs

2 The Navaho, Cambridge, Massachusetts, 1946, cité par Bertrand de Jouvenel (1977, p. 191). 
(Baechler [1994], Silberbauer [1982]); il était également l'unique forme de décision collective légitime dans les communautés villageoises en Kabylie (Mahé [2000]), en Afrique noire (Abélès [2003], Terray [1988]) et en Asie (Popkin [1979], Smith [1959]). Les communautés villageoises européennes du Moyen Age avaient également l'usage des assemblées délibératives arrêtant leurs décisions sans vote, notamment dans le centre et le Nord de l'Europe: Otto Gierke (cité par Dumont [1983], p. 99) a noté la prévalence de l'unanimité pour l'Europe germanique; l'assemblée des chefs de clans islandais, Althing, fonctionnait sans doute de la même manière (Byock [2001]). Le souci du consensus prédominait encore dans les décisions au sein de certains villages scandinaves il y a trente ou cinquante ans (Yngvesson [1978] pour la Suède, Barnes [1954] pour la Norvège).

Par ailleurs, les techniques de vote n'ont été pratiquées que tardivement dans la plupart des sociétés, et notamment via la modernisation dont l'occident fut l'initiateur puis le diffuseur. L'Europe occidentale médiévale n'avait pas, semblet-il, la mémoire des techniques électorales de l'antiquité grecque et romaine. Et c'est principalement au sein des ordres monastiques et du clergé que ces techniques furent redécouvertes (Moulin [1958], Gaudemet [1979]) ${ }^{3}$, puis transmises aux assemblées politiques provinciales. La colonisation puis la décolonisation ont été les principaux vecteurs de la diffusion de la pratique du vote, y compris au niveau des villages, en Afrique et en Asie.

Bref, le consensus ou l'unanimité semblent avoir été le mode de décision collective prédominant dans la quasi-totalité des sociétés humaines, avant que le vote ne tende à le remplacer. Pourtant, les descriptions proposées de ce qui est appelé décision par consensus ou décision à l'unanimité sont le plus souvent décevantes (1.1). Rares sont celles qui rendent compte précisément de quelle manière ce consensus ou cette unanimité sont constatés par les participants (1.2). Or, la description de la manière par laquelle la décision collective est arrêtée permet de prendre conscience de l'usage fréquent, y compris dans nos sociétés modernes, de ce mode de décision (1.3).

Une précision s'impose ici, avant d'examiner le problème de la description des décisions collectives. Certaines descriptions peuvent être à tort jugées insuffisantes, parce que le lecteur y cherche ce qu'il ne peut trouver. L'absence de description précise des décisions collectives, par exemple dans certains travaux ethnologiques, ne tient pas toujours au faible intérêt de l'ethnologue pour la chose. Cette absence est parfois le reflet de ce que la notion même de décision collective n'est pas pertinente pour la société qu'il étudie. En effet la pertinence de cette notion suppose que la décision prise collectivement génère une obligation, lie les membres en faveur du respect de sa mise en œuvre. Or, certaines sociétés, notamment amérindiennes, ne connaissent pas un tel phénomène. Les observations de Catherine Alès sur les prémisses des raids collectifs contre des ennemis, chez les Yanomami, montrent que la succession de prises de paroles des chefs de foyer qui précède l'expédition ressemble mais ne s'apparente pas à la préparation d'une décision collective. Malgré l'interdépendance des membres d'un groupe de maisonnées voisines - et le fait que tout le monde, participants ou non au raid, risque

3 Le vote à la majorité était également présent dans les monastères du japon médiéval (Souyri [2003]) et plus généralement dans les monastères bouddhiques (Dumont [1983], p. 36). 
de pâtir de ses conséquences -, personne ne se sent lié par cette succession de prises de parole (Alès [2006]). Celle-ci s'apparente à une consultation, permettant à chacun d'évaluer ce qu'il sera possible d'entreprendre. Dans une telle société, la coordination et l'interdépendance constantes et fortes se passent de décision collective. Cette observation permet en retour de penser qu'une partie des usages de la règle d'unanimité mentionnés par les historiens et les ethnologues ne correspond sans doute pas à de véritables décisions collectives, mais à des assemblées permettant de constater les coalitions possibles, point que Simmel avait vu à sa manière dans son excursus sur la règle de majorité (1999).

Il va de soi que s'il n'y a pas de décision collective, il ne saurait y avoir de problème de sa description. Les pages qui suivent ne concernent donc que des sociétés et des situations où la décision est collective pour trois raisons: ses conséquences concernent la collectivité, restreinte ou non aux participants à la décision; elle fait l'objet d'une travail collectif des participants (ni tirage au sort, ni délégation à un «dictateur» au sens de Arrow [1974]); la décision collective lie les membres du groupe considéré.

\subsection{La décision à l'unanimité ou par consensus}

Il est possible de généraliser le propos de David Turton à la quasi-totalité de la littérature ethnologique, du moins à tous les travaux que j'ai pu consulter: "Il est une caractéristique frustrante des écrits sur les sociétés pastorales de l'Afrique de l'Est: même quand elles s'intéressent explicitement à des sujets tels que le leadership et le contrôle social, elles contiennent généralement peu d'information sur une activité qui apparaît, dans ces mêmes écrits, comme un trait marquant de la vie publique de ces sociétés, la tenue d'assemblées pour discuter et formuler des décisions qui concernent toute la communauté » (Turton [1975], p. 163). En tenant compte des progrès réalisés depuis ce constat et en centrant le diagnostic sur ce qui m'intéresse ici, je dirais que les travaux sur les assemblées peuvent être désormais forts riches (Detienne [2003]) tout en étant peu précis sur la prise de décision, l'essentiel de la description et de l'analyse des auteurs portant sur la sélection des participants, le déroulement des sessions, les rituels et leur signification. Aussi, alors même que l'usage du consensus ou de l'unanimité est presque toujours mentionné, la manière exacte dont ce consensus ou cette unanimité se forment et surtout permettent d'arrêter la décision collective est très rarement décrite. Ce problème de description a au moins trois ressorts: la difficulté d'un recueil de données suffisamment précises; le faible intérêt des ethnologues pour la prise de décision qui n'occupe qu'une partie très restreinte de leurs observations; la prévalence intellectuelle du modèle du vote. Je mentionnerai deux études remarquables pour illustrer ce point.

Le livre de Samuel Popkin (1979) est une référence majeure pour l'application du paradigme de l'individualisme méthodologique à l'étude des sociétés paysannes. Il y précise que jusque dans les années 30 et 40, dans les villages du Sud Vietnam, les décisions collectives étaient prises à l'unanimité; et il explique la prévalence de cette règle de décision par le système d'interdépendance dans lesquels sont pris les villageois: une décision collective peut avoir des conséquences dramatiques pour une famille, d'où le caractère adapté d'une règle qui accorde à chacun un droit de veto, tandis que la division sexuelle du travail permet d'absorber 
le temps dépensé pour se mettre d'accord (les hommes bavardent pendant que femmes et enfants travaillent). Quel que soit l'intérêt de l'analyse proposée, il faut noter que l'ouvrage, qui utilise des données de seconde main, ne propose aucune description du fonctionnement des conseils de village: le lecteur ne peut donc savoir si l'unanimité requise est attestée par un vote ou par un autre procédé.

L'étude de Marc Abélès sur les assemblées dans la société Ocholo, en Ethiopie méridionale, offre au lecteur une description beaucoup plus riche du processus de décision collective. Ici l'auteur précise que le vote est absent: sa description s'appuie sur le contraste entre le vote majoritaire qui nous est familier et une décision qui exige un consensus ou une unanimité (les deux notions étant confondues) sans vote. Mais le lecteur ne sait pas comment l'unanimité-consensus est révélée aux participants. La citation d'un extrait est nécessaire pour saisir la subtilité du problème de la description:

«Quand il juge que la réunion a suffisamment avancé, le grand dignitaire tente de syn-
thétiser les débats et d'émettre une proposition qui facilite la prise de décision. Il importe
qu'une unanimité se dégage au terme des délibérations. Au fil des interventions prend
corps une véritable opinion dominante qui s'affirme au point d'emporter tous les suf-
frages. Il n'y a pas de vote, il n'est pas question de compter les voix. Il faut que le consen-
sus se produise, faute de quoi il apparaît préférable de clore l'assemblée et de reprendre
le débat par la suite. ... L'assemblée n'équivaut pas à une sommation d'individus-
citoyens. C'est ce qu'exprime clairement le mode de détermination du consensus, où il
n'est pas question d'additionner des voix. Ce qui importe avant tout, c'est de dégager une
véritable unanimité.» (Abélès [2003], p. 400 et 404).

La description fine de Marc Abélès butte ici sur une sorte de contradiction. D'un côté, il souligne que la pensée politique des Ocholos n'est pas celle d'un individualisme politique, l'assemblée n'est pas conçue comme le rassemblement de citoyens et la légitimité de la décision collective n'est pas garantie par la sommation des parcelles individuelles de souveraineté. D'un autre côté, on ne voit pas comment pourrait être constaté l'achèvement de la formation du consensus autrement que de manière additive, puisque ce consensus est identifié à une « véritable unanimité». L'expression semble indiquer qu'il y a consensus quand tout le monde approuve la même option. Comment les Ocholos constatent-ils qu'une option fait l'unanimité? Il est remarquable qu'Abélès utilise une métaphore électorale («emporter tous les suffrages») au moment même où il souligne que le consensus se réalise sans vote. Mais comment constater l'unanimité sans observer que chacun des participants a exprimé de quelque manière (main levée, hochement de tête) son approbation à l'égard de la même option, soit bien par une forme de vote? Le constat de l'unanimité impose une sorte de vote. Aussi l'assimilation du consensus à l'unanimité paraît-elle contradictoire avec l'observation de l'absence de vote.

Ainsi Popkin oppose-t-il l'usage de la règle de majorité à celle de l'unanimité, sans pouvoir préciser si cette unanimité est attestée dans les villages Sud vietnamiens par un vote. Tandis qu'Abélès insiste sur le contraste entre vote et consensus, mais l'assimilation de ce consensus à une unanimité rend difficilement compréhensible l'absence d'un vote, aussi informelle que puisse être l'expression des opinions. Dans les deux cas, même si c'est à des degrés très différents, la description est insuffisante. Il manque une partie du processus de décision collective, pourtant essentielle: sa terminaison, la manière par laquelle la décision est arrêtée. 


\subsection{Un mode d'arrêt de la décision collective}

Le problème de description des travaux mentionnés précédemment tient à ce qu'ils ne permettent pas de répondre à la question suivante. La décision par consensus correspond-elle à un mode d'arrêt de la décision différent de celui observé dans les procédures de vote? Deux études nous permettent de répondre très exactement à cette question.

\section{La décision par consensus dans un village du Soudan}

L'étude de Sherif El-Hakim sur les processus de décision dans un village du Soudan, observés en 1970 et 1971, fait exception dans la littérature ethnographique par son attention pour la procédure d'arrêt collectif de la décision ${ }^{4}$. Les deux mille habitants de Khuriet, généralement issus de la même ancienne tribu nomade arabe, vivent pour la plupart de petites cultures et de l'élevage de chameaux, de moutons et de chèvres. Le village est composé de dix hameaux entourant une aire centrale comprenant le court du Sheikh, la place du marché, le centre de distribution d'eau, un dispensaire et deux mosquées. La participation aux décisions touchant la collectivité est régie par deux principes. Il y a d'abord des «notables» ou des personnages disposant d'une certaine autorité, celle-ci étant souvent associée à des responsabilités. Ces notables ont un rôle et un poids spécifique dans les décisions collectives. Le deuxième principe est que tout villageois a le droit de participer dès lors que la décision touche ses intérêts. Là où leurs intérêts sont engagés, les villageois estiment, et se voient reconnaître, le droit de participer. Seuls les hommes, constamment impliqués dans divers conciliabules pendant que femmes et enfants assurent l'essentiel de l'activité productive du village, participent aux fréquentes et longues réunions du village. L'initiative d'une réunion en vue d'une décision collective ne peut être le fait de n'importe quel villageois, mais seulement des plus influents d'entre eux. L'annonce d'une telle réunion se fait par bouche à oreille. On prend soin de faire en sorte que les gens concernés par les décisions à prendre soient informés et puissent être présents à la réunion. Les gens s'acheminent alors vers la place principale, par petits groupes de deux ou trois, et discutent les uns avec les autres en attendant que le nombre des présents s'accroisse. Cette période d'attente peut durer une heure et cesse quand un notable prend la parole et présente longuement le but de la réunion, les données du problème qu'elle doit traiter, les opinions émises lors des réunions informelles antérieures. Cela se fait dans le plus grand silence, sauf si quelqu'un souligne l'absence d'un villageois concerné, ce qui exige une discussion pour savoir si cette absence est dommageable ou pas. Enfin, celui qui a pris la parole conclut son discours en exposant ce qui lui semble être la solution. De nombreuses voix s'élèvent en même temps, certains donnent leur opinion au groupe qui discute à proximité, d'autres crient et gesticulent pour attirer l'attention de tous. Au bout d'un certain temps, pendant lequel l'agitation et le brouhaha s'apaisent puis s'élèvent plusieurs fois, le silence se fait pour écouter un homme, toujours différent de celui qui avait ouvert la réunion, qui présente ce qui lui semble être le consensus dégagé par la discussion. Trois cas de figure sont alors envisageables.

4 Cette exception est sans doute à mettre en relation avec l'usage de la théorie de la décision collective de Coleman (1966), référence rarement mobilisée en ethnologie. 
- Si à la suite de cette proposition de consensus, quelques manifestations d'acquiescement sont entendues, sans autres complications, même si ce soutien ne vient que d'un ou deux participants pendant que tous les autres restent silencieux, la réunion prend fin et la proposition énoncée devient la décision collective.

- Dans les rares cas où la proposition est explicitement contestée, invectives et brouhaha rompent la réunion.

- Le plus souvent, si des désaccords sont exprimés, c'est de manière détournée. Ils sont manifestés indirectement par l'introduction de contre-propositions qui ont peu de relation avec le problème en discussion et qui contribuent à transformer l'enjeu de la réunion. Alors l'assemblée reprend son allure bruyante et désordonnée jusqu'à ce qu'une autre proposition, sensée exprimer un nouveau consensus, soit avancée de la même manière que la première fois. Si de nouvelles propositions ne peuvent émerger ou si elles sont successivement rejetées, le temps passant, la réunion s'achève sans décision arrêtée.

\section{Les décisions sur les bateaux de pêche suédois}

Dans les assemblées que nous venons de décrire, aucune technique de décompte des opinions n'est perceptible, aucune règle formelle d'agrégation des avis n'est utilisée. Barbara Yngvesson (1978) a noté la même absence dans les décisions qu'elle a observées en 1967 et en 1968 dans une communauté de pêcheurs, sur une petite île de la côte ouest de la Suède. Au sein des bateaux de pêche comme au sein de l'assemblée qui tient lieu de corps politique de l'île, règne le même souci égalitaire. Les décisions collectives y suivent le même protocole. Quand une décision critique doit être prise lors d'une sortie en mer, par exemple changer de lieu de pêche, aucun membre de l'équipage ne saurait, fût-il l'un des propriétaires du navire, imposer un choix. La décision doit être collective. Celle-ci respecte trois étapes: 1) l'un des pêcheurs propose un changement de localisation, sous la forme d'une suggestion; 2) suit une période de temps d'au moins une demi-heure où des réactions sont attendues; 3) en l'absence de contre-propositions, le même homme réitère sa proposition et le bateau rejoint la zone annoncée. Le conseil de l'île respecte ces trois étapes, à partir des propositions de son président de séance.

Les deux monographies de El-Hakim et d'Yngvesson proposent une description précise de la manière dont est arrêtée la décision collective. La discussion n'est pas suivie par un vote, mais par l'émission d'une proposition ou d'une série de propositions censées correspondre à un consensus. Celui-ci n'est pas attesté par un dénombrement des opinions, mais par le constat d'une absence d'opposition à la dernière proposition émise.

\subsection{Inventaire d'un usage}

Pour des raisons explicitées plus loin, je propose d'appeler «décision par consensus apparent» ce mode de décision collective. Une fois que l'on a à l'esprit ses caractéristiques, il apparaît que son usage est plus fréquent qu'on ne l'imagine. Pour ma part, les lectures et les enquêtes que j'ai pu réaliser sur quelques assemblées délibérantes, m'invitent à distinguer trois contextes-types de son occurrence: 1) dans les sociétés étudiées par les ethnologues, pour simplifier, je 
rassemblerai ces emplois sous le terme consacré de palabre; 2) dans des aréopages, le terme étant pris ici au sens moderne d'assemblée de personnes éminentes, sages, savants, magistrats, réunis au nom de leur compétence; 3) dans des assemblées ou commissions qui utilisent plusieurs règles d'arrêt de la décision, parmi lesquels la décision par consensus qui, le plus souvent, est employée comme un expédient.

La palabre. Sherif El-Hakim et Barbara Yngvesson décrivent précisément la séquence négligée par les descriptions proposées par les autres ethnologues: celle de l'arrêt de la décision. On peut se demander si les nombreux auteurs qui ont évoqué des décisions à l'unanimité ou par consensus n'avaient pas en fait affaire à ce que j'appelle désormais la décision par consensus apparent. Il s'agit d'une conjecture que je ne peux ici transformer en affirmation démontrée. Mais elle me paraît d'autant plus plausible que plus les descriptions proposées par les ethnologues sont précises, plus elles se rapprochent de la description complète de l'arrêt de la décision par consensus apparent. Plus une description est précise, plus longue est la liste des caractéristiques décrites. L'ordre de mention des traits est à peu près le suivant, par degré de précision croissant: 1) décision à l'unanimité ou au consensus; 2) affirmation de l'absence de vote; 3) le consensus n'équivaut pas à l'unanimité réelle mais au consentement des réticents minoritaires; 4) des propositions de consensus facilitent la décision; 5) il y a décision quand une proposition de consensus n'est plus contestée. Ainsi l'analyse secondaire des descriptions que j'ai pu rassembler me semble donner une bonne plausibilité à l'affirmation selon laquelle la description la plus complète de la palabre est celle de Sherif El-Hakim ${ }^{5}$.

Quoiqu'il en soit, on comprend aisément que ce mode convienne à de petites sociétés marquées par une assez forte autarcie et surtout par une très forte interdépendance des intérêts individuels et où, de surcroît, une partie de la population, en l'occurrence les hommes adultes, peut consacrer tout son temps aux conciliabules nécessaires à ce mode de décision. Aussi pourra-t-on penser que cette règle de décision n'est pas observable dans nos sociétés. Il n'en est rien. Les deux autres usages que je vais mentionner maintenant se rencontrent dans les sociétés modernes.

Les aréopages. Le vote est le modèle dominant des décisions collectives dans les sociétés occidentales, et ce bien au-delà des seules élections politiques. Pourtant, on y rencontre des organes délibératifs qui rejettent la possibilité d'arrêter leur décision par un dénombrement des opinions. Le vote est utilisé exceptionnellement, comme dernier recours, si les participants ne parviennent pas à un consensus. C'est le cas de certaines cours constitutionnelles (Ferejohn, Pasquino [2002]; Pasquino [2006]) et de certaines commissions d'experts comme celles qui ont la charge d'autoriser la mise sur le marché des médicaments en Europe et en France (Hauray [2005], Urfalino [2006]). Les membres de ces aréopages, non élus et dépourvus de fonction représentative, sont nommés pour leur compétence. Il importe de noter que, comme le souligne Pasquino (2006), les décisions en cause ne sont pas réductibles à l'alternative oui/non. Face à la question de la constitu-

\footnotetext{
5 Yngvesson, précise sur l'arrêt, est beaucoup plus discrète que El-Hakim sur la discussion.
} 
tionnalité d'une loi, les arguments qui justifient la réponse des juges sont aussi, voire plus, importants que son contenu positif ou négatif. De même, face à une demande d'autorisation de mise sur le marché d'un médicament, la réponse n'est pas simplement «oui» ou «non», mais «non» tant que telles conditions ne seront pas remplies ou «oui» dans telles conditions très précises. Dans les deux cas, la décision est arrêtée quand une proposition finale, correspondant en fait à un paquet de choix et d'attendus, ne suscite plus d'objections ${ }^{6}$.

Dans le cas de la palabre, aucun autre mode de décision n'est utilisé. Soit les sociétaires ne connaissent pas les pratiques du vote, soit ils les connaissent mais les estiment inadaptées à leur société (Terray [1988]; Ferme [1998]). De la même manière, dans les aréopages, le recours au vote est exceptionnel. Pourtant, il est des cas où la décision par consensus est utilisée dans des assemblées et des commissions délibérantes qui par ailleurs pratiquent régulièrement le vote. Voici trois exemples correspondant à ce type d'usage.

Le consensus comme expédient. Le premier exemple est emprunté à une étude sur les Fonds Régionaux d'Art contemporain, créés en France en 1982. Le conseil d'administration de ces fonds avait la charge d'accepter ou de rejeter les propositions d'achats d'œuvres d'art contemporain faites par un comité d'experts. Dans une partie des régions françaises, la majorité des élus régionaux qui le composaient était hostile aux choix des experts en faveur d'œuvres déroutantes pour des néophytes; en revanche, le président du conseil d'administration de ces FRAC, souvent un important notable politique régional, était plus enclin à suivre l'avis des experts, moins par goût que parce qu'il attendait de la collection de niveau international que les experts souhaitaient constituer un bénéfice pour la renommée de la région. Dans l'une des trois régions étudiées, le président du FRAC avait l'habitude, après que les œuvres avaient été présentées et au moment où l'on pouvait s'attendre à un vote, de dire ceci avec assurance aux autres administrateurs: «Chers amis, vous avez écouté les experts, les œuvres proposées sont de grande qualité. Nous n'allons quand même pas voter! Je propose qu'on les prenne toutes, vous êtes d'accord?». Les élus réticents n'osaient pas s'opposer à l'homme politique montant de la région, le président obtenait sans vote, et donc sans l'expression de désaccords, l'achat des œuvres. Toutefois, il veillait à ne pas susciter un rejet brutal de son stratagème et s'il sentait - ou si l'un de ces conseillers avait senti - une opposition trop forte à une œuvre, il consentait à la sortir du lot à acheter (Urfalino, Vilkas [1995]).

J'ai pu observer de près pendant quatre années le fonctionnement d'une section du Comité national de la recherche scientifique, principal dispositif d'évaluation scientifique en France. Chaque section, composée d'une vingtaine de membres élus ou nommés, assure l'évaluation des laboratoires et des chercheurs du Centre National de la Recherche scientifique. Toutes les décisions collectives dont la section a la charge (classement des laboratoires, des chercheurs candidats à une promotion ou à un recrutement) sont prises avec des machines à voter et

6 Il importe de préciser que d'autres aréopages ayant les mêmes tâches pratiquent le vote: il en va ainsi des cours suprêmes des Etats-Unis et, pour les médicaments, des comités de la Food and Drug Administration. On ne peut aborder ici la réflexion sur ces différences. 
selon la règle de majorité. Pour autant, certaines décisions a priori peu importantes et semblant susciter aisément la convergence des opinions, étaient arrêtées par consensus apparent. C'était le cas des décisions de procédure, sortes de décisions de deuxième rang dont l'objet était de fixer la manière de prendre les décisions de premier rang: le meilleur exemple étant le choix du moment où l'assemblée peut clore le débat sur une question et trancher par un vote. Ainsi, après une longue discussion, le président de la section pouvait déclarer: "Chers collègues, il me semble que nous avons suffisamment discuté, je vous propose de passer au vote». Si personne ne contestait l'invitation, la proposition valait décision et nous passions au vote. Il arrivait parfois qu'un membre de la section demande la prolongation du débat. Celui-ci reprenait jusqu'à ce que le président reprenne sa proposition de passer au vote. Deux ou trois fois, la durée de la discussion fut l'objet d'un désaccord prolongé, aussi le président de la section proposa-t-il que l'on vote pour départager ceux qui souhaitaient la poursuite du débat et ceux qui voulaient passer au vote. On vota donc pour décider de voter ou non ${ }^{7}$. Remarquons cependant que pour ce vote au carré, la décision de voter pour savoir si l'on allait voter fut prise selon la règle du consensus apparent! On touche ici à une sorte de limite logique du formalisme électoral: sauf régression indéfinie, il faut bien un autre mode de décision que le vote pour que le groupe décide collectivement de passer au vote.

Le troisième exemple, le plus documenté, est issu d'une étude minutieuse et systématique des décisions prises au sein d'un parti politique suisse dans le canton de Berne (Steiner, Dorff [1980]). Utilisant l'interview, l'observation et l'analyse des documents, Steiner et Dorff ont suivi 111 réunions de ce parti, de janvier 1969 à septembre 1970. Ils y ont observé 466 situations où une décision devait être prise, suite à un désaccord sur une action à entreprendre. Ils avaient prévu trois modes de règlement de ces situations : par un vote à la majorité; par la formation d'un accord explicite et oral après que les partisans d'une option se sont clairement ralliés à l'autre option; par la non décision. 37\% des 466 cas n'entraient dans aucune des trois catégories envisagées. Les auteurs ont d'abord songé à une catégorie résiduelle. Puis, ils ont dû admettre que ces cas renvoyaient à un quatrième type correspondant à la séquence suivante: après un temps de discussion, un membre du groupe donne une interprétation des conclusions qui, selon lui, ressortent du débat. Si personne ne s'oppose à cette interprétation, celle-ci vaut décision. Grâce à l'observation fine des réunions et des conciliabules qui s'ensuivent, les auteurs ont pu estimer que, sur les 170 cas de décision par consensus apparent, 85 avaient une issue conforme à la majorité des opinions, 19 à une minorité, tandis que 66 dénouaient une situation sans majorité détectable. Deux des interrogations de Steiner et Dorff face à ces résultats nous intéressent plus particulièrement. Pourquoi ceux qui continuent à désapprouver la position qui bénéficie de l'«interprétation » ne manifestent-ils pas leur désaccord? Pourquoi ne réclament-ils pas un vote, notamment pour s'assurer qu'ils sont bien minoritaires? La réponse issue de leurs

7 Le redoublement de la procédure, voter pour décider de voter ou non, peut paraître ridicule, Uderzo en fit l'objet d'un gag dans l'un de ses albums d'Astérix (Goscinny, Uderzo, 1991, p. 22). Pour autant, la fixation de la durée de la délibération précédant le vote peut être un enjeu important, voir notamment dans le cas du CNRS (Vilkas [1996]). 
interviews et observations est la suivante: Ceux qui désapprouvent l'«interprétation» qui vaut décision - et qui pourtant ne la contestent pas - n'ont pas une idée précise de la réelle distribution des préférences, mais ils se donnent peu de chance d'obtenir la majorité au cas où ils réclameraient un vote. Aussi préfèrent-ils ne pas rendre patent leur échec et/ou souhaitent ne pas renforcer, par un vote gagnant, la légitimité de la position qui l'emporte par «interprétation».

Dans ce dernier cas, l'usage de la décision par consensus apparent a plus de légitimité que son utilisation comme un coup de force, comme dans le cas du FRAC, et n'est pas réservé à des aspects techniques et sans grand enjeu de la décision, comme dans le cas du CNRS. Utilisée concurremment avec d'autres modes, la décision par consensus apparent est cependant moins légitime que le vote. Et elle est susceptible d'être rejetée au bénéfice de ce dernier quand les enjeux sont importants et que l'assemblée est fortement divisée. Dans la mesure où tout participant est en droit de réclamer un vote, la décision par consensus apparent est retenue dans deux situations: 1) elle semble accélérer une décision dont le résultat est estimé conforme au vœu de la majorité; 2) elle correspond à un équilibre des anticipations de celui qui propose une interprétation en espérant qu'elle ne sera pas contestée et de ceux qui, tout en la rejetant, préfèrent se taire parce qu'ils pensent être minoritaires et ne souhaitent pas que cette minorité soit révélée.

A l'aide des études et des observations inventoriées, il est maintenant possible d'examiner les propriétés de ce mode de décision.

\section{LES PROPRIÉTÉS CONSTITUTIVES DE LA DÉCISION PAR CONSENSUS APPARENT}

L'ensemble des cas et des descriptions que j'ai évoqués et parfois résumés dans la partie précédente permet de dégager une règle de décision spécifique, dont la différence avec les différentes formes de vote ne me semble pas avoir été suffisamment bien estimée. Je propose de nommer décision par consensus apparent cette règle de décision spécifique, en référence à ses six caractéristiques constitutives:

- C'est une règle d'arrêt de la décision, au même titre que les procédures de vote;

- C'est la constatation collective d'un consensus apparent qui tient lieu de règle d'arrêt;

- Elle ménage une impression de continuité entre processus et arrêt de la décision;

- Cette règle prévaut dans un contexte où, pour quelque raison, la recherche du consensus est indexée sur le souci de la qualité de la décision;

- Le consensus apparent exige non pas l'unanimité mais, à côté de ceux qui approuvent, le consentement des réticents;

- La contribution des participants à la décision est marquée par le contraste entre un droit égal à la participation et une inégalité légitime des influences. 


\subsection{Une procédure d'arrêt}

La notion de règle de décision mérite une précision dans la mesure où de nombreuses règles de natures très différentes sont susceptibles d'intervenir dans des décisions collectives : celles qui régissent l'initiative du processus, la sélection des participants, l'ouverture et la fermeture des débats, etc. (Richards, Kuper [1971]). Le problème de description évoqué au début de cet article ne concerne qu'une partie de l'ensemble du processus de décision: sa terminaison par un arrêt collectif d'une intention d'agir. C'est sur ce point très précis que les monographies de Sherif El-Hakim et de Barbara Yngvesson tranchent par leur minutie avec les autres descriptions. Aussi, dans cet article, la notion de règle de décision est-elle employée dans le sens restreint de règle d'arrêt collectif de la décision.

On peut conjecturer que les difficultés de description observables dans la littérature ethnologique tiennent d'abord au fait que les chercheurs occidentaux ont une conception de la décision collective fortement influencée par la connaissance des techniques électorales. Celles-ci servant de référence, les occurrences de la décision par consensus apparent sont assimilées à des décisions à l'unanimité réalisées de manière informelle. Par ailleurs, les imprécisions conceptuelles attachées à l'usage du terme «décision» n'encouragent pas la détection d'une règle d'arrêt quand, faute d'un vote, rien n'en dispense pas la présence familière. Polysémique dans son usage courant, désignant aussi bien le processus que le résultat, le terme de décision n'a pas été beaucoup mieux défini par les sciences sociales. La sociologie des organisations, les études de management et la science politique ont concentré leurs réflexions sur le processus de décision, entendu comme l'ensemble des séquences et mécanismes contribuant à la perception d'un problème à résoudre, à la production d'options alternatives et au choix de l'une d'entre elles. Soucieuses de démythifier le volontarisme souvent attaché à l'idée de décision, elles ont à juste titre mis en cause l'idée d'un «moment» de la décision et enfin l'absorption de la détermination de la décision dans la personne du «décideur». D'où leur faible intérêt pour le problème de l'arrêt de la décision. Ce faisant, elles ont considérablement amélioré notre compréhension des processus de décisions, mais ont laissé dans une certaine indétermination le concept même de décision: elles ont ignoré le concept de délibération et ont assimilé les notions de choix et de décision (Urfalino [2005]). Pour sortir de cet embarras, il importe d'abord de constater que le concept de décision appartient au registre de l'action. L'objet d'une décision n'est jamais un état de la connaissance (on ne décide pas que tel énoncé est vrai ou faux au regard de ses référents), ce que l'on décide, c'est une action à entreprendre. Ensuite, toute action intentionnelle n'est pas précédée d'une décision. Mais, pour qu'il puisse être dit qu'il y a eu décision, il faut pouvoir distinguer un laps de temps entre cette décision et sa mise en œuvre (Raz [1975]). Ce laps de temps pointe une distinction conceptuelle qui livre la nature de la décision: elle est l'arrêt d'une intention d'agir. La décision proprement dite n'est ni l'ensemble d'un processus, ce qui concourt à la décision, ni ce qui en résultera, l'action qui a été décidée: il y a décision quand entre la délibération, d'un côté, et l'action, de l'autre côté, s'interpose l'arrêt d'une intention. Une clarification du concept de décision amène ainsi à mettre en avant la notion d'arrêt de l'intention d'agir.

Or dans le cas d'une décision individuelle, il n'est pas rare que l'arrêt ne soit pas distinguable de la fin des opérations mentales, la délibération et le choix. Il en 
va tout autrement dans le cas d'une décision collective, au sens restreint où j'emploie ce terme: c'est-à-dire une décision en assemblée, faisant l'objet d'un travail collectif et sur laquelle chacun des participants co-présents a la possibilité d'intervenir et de peser directement. Ainsi entendue, la décision collective suppose une procédure d'arrêt collectif.

Or une telle procédure doit satisfaire deux exigences pratiques : il faut bien sûr que puisse être déterminé un arrêt de la décision (exigence de fixation); mais il faut également que cet arrêt soit produit, perçu et accepté collectivement, par tous et par chacun (exigence de reconnaissance mutuelle de la fixation). Cette deuxième exigence induit une contrainte de description pour l'observateur qui souhaite rendre compte de ce mode de décision collective: la description doit avoir une dimension phénoménologique (au sens premier de ce qui apparaît). Elle doit montrer de quelle manière, par quel procédé, il apparaît à tous les participants qu'un arrêt a émergé de leurs contributions au processus. En effet, dans la mesure où les êtres humains ne sont pas mutuellement transparents et que la communication réciproque de leurs pensées suppose l'usage de techniques d'expression, il faut que les membres aient les moyens de savoir sans ambiguïté si et quand la décision est prise ${ }^{8}$. Or, de deux choses l'une: ou bien le consensus est l'unanimité et il doit être rendu perceptible par une technique d'expression des opinions permettant de constater qu'il y a bel et bien unanimité - on est alors en présence d'une technique électorale qui peut-être très sommaire (hochement de tête par exemple); ou bien il n'y a pas une telle technique d'expression et le décompte des opinions est impossible. Dans ce dernier cas, c'est l'absence manifeste d'opposition à une proposition qui transforme celle-ci en décision: l'arrêt se fait par le constat d'une absence.

La décision par consensus apparent et le vote sont donc deux procédures distinctes d'arrêt de la décision collective. L'une ne comprend pas plus que l'autre l'ensemble du processus de décision, mais l'une et l'autre régissent sa terminaison. Elles le font d'une manière radicalement différente, comme en atteste l'examen de la notion de consensus apparent.

8 Il peut arriver qu'un ou plusieurs participants n'aient pu observer l'arrêt et que celui-ci fasse pour eux l'objet de conjectures ou de croyances individuelles. Pour autant, qu'une décision ait été prise, ou que l'on soit en train de la prendre, ou que finalement on ait renoncé momentanément à arrêter une option, ce sont là, la plupart du temps, autant de faits, c'est-à-dire d'états ne prêtant pas à discussion. La règle de décision instituée permet de reconnaître ces états. Ainsi conçue, la décision correctement prise ne me semble pas pouvoir être assimilée à une croyance collective, comme le suggère Margaret Gilbert dans l'un de ses textes. Elle illustre sa conception de la croyance collective en décrivant un cas imaginaire et plausible de discussion des membres d'un collège d'Oxford sur la qualité des repas servis à leur «Table Haute». Sa description correspond exactement à ce que j'appelle décision par consensus apparent, à une différence importante près: elle n'envisage pas, ou du moins ne précise pas, que la terminaison de la discussion qu'elle imagine puisse avoir le statut d'une décision, soit l'arrêt d'une intention d'agir. Il est pourtant difficile de ne pas y penser à la lecture de cet extrait: « le plus ancien des membres élève la voix et déclare sur un ton catégorique et définitif: "Il est clair que ce n'est pas assez varié! Il faut que quelqu'un aille parler au chef !" Il regarde autour de lui brièvement. S'ensuit une pause. Puis: "Si nous passions à un autre sujet?" Personne ne fait d'objections.» (Gilbert, 2003, p. 96). Discuter la thèse de Gilbert exigerait une clarification du statut des concepts de décision, de règle de décision et de croyance collective que je ne peux entreprendre ici. 


\subsection{Apparence de consensus et consensus apparent}

L'adjectif «apparent» doit ici être compris en gardant à l'esprit ses deux sens. Il peut être entendu comme le terme s'opposant à réel; ce qui est apparent est susceptible d'être faux ou trompeur. Il renvoie également à ce qui apparaît, ce qui se présente à nous et devient saisissable: «apparent» fait alors écho au sens premier, grec, de «phénomène».

Le consensus est d'abord apparent au sens où l'on peut douter de sa réalité. Cela résulte de ce qu'il n'est pas attesté par un décompte des opinions, mais par l'absence de contestation à une proposition. Si bien qu'une fois la décision prise, la distribution exacte des préférences peut être méconnue. Ce point distingue clairement la décision par consensus apparent de ce que serait une décision à l'unanimité proprement dite. Celle-ci suppose une technique de décompte des avis ou à tout le moins la possibilité de constater que chaque participant approuve la même option: selon une telle règle, la décision est prise quand tous les avis sont formellement convergents ou identiques. A l'opposé, la décision par consensus apparent exige non pas l'unanimité ou la quasi-unanimité, comme le sens courant du mot consensus incline à le penser, mais l'absence de désaccords exprimés; or cette absence laisse pendante la question de savoir si les silencieux approuvent ou désapprouvent. La double négation, «il n'existe pas d'expression d'un refus de la proposition $\mathrm{P}$ », n'équivaut pas à l'affirmation «tout le monde exprime son approbation de $\mathrm{P} »$ ?

Le consensus est ensuite apparent au sens où il apparaît. La décision par consensus apparent suppose deux choses: l'énoncé d'une proposition et la constatation qu'elle n'est pas rejetée. Le caractère patent de l'absence d'expression de désaccord est essentiel: il fait en sorte que la décision puisse être collectivement arrêtée. Un temps d'arrêt suit cette proposition, permettant de constater la contestation ou son absence accompagnée de quelques approbations. Il faut souligner à nouveau l'importance de la négation. Ce qui apparaît, ce n'est pas directement le consensus, mais l'absence de dissensus manifesté. Ce qui importe, c'est que cette absence, valant attestation du consensus, puisse faire l'objet du même constat par chacun des participants à la décision collective: chacun constate qu'une proposition d'action à entreprendre censée résulter de la discussion ne fait pas l'objet de contestation explicite; ce faisant, cette synthèse devient la dernière proposition et la décision arrêtée.

\subsection{La continuité entre délibération et décision}

Lorsqu'une proposition est émise, elle est susceptible soit de valoir décision, si personne ne la conteste, soit de n'être que l'une des étapes de formation de la proposition finale, si elle est contestée. La phase finale du processus de décision n'est pas établie à l'avance. Le fait qu'une proposition soit la dernière et vaille décision n'est su que rétrospectivement, quand on a constaté l'absence d'opposition.

Cet aspect est, après l'absence de dénombrement des avis, une deuxième grande différence avec l'arrêt de la décision par vote. Dans ce dernier cas, il y a discontinuité entre la délibération, entendue comme la discussion collective en

\footnotetext{
9 La sincérité de l'expression de l'accord ou du désaccord importe peu ici.
} 
vue de la décision, et l'usage de la technique électorale. On a alors affaire à deux moments bien séparés, au point même, comme on l'a noté, qu'il est nécessaire de décider ou de se référer à une règle pour savoir quand la discussion doit laisser place au vote. L'arrêt par consensus apparent assure au contraire une continuité entre la délibération et la décision. La succession des propositions non retenues entre plusieurs phases de discussion, d'une part, et le fait que le moment et le statut de dernière proposition ne puissent être définis à l'avance, d'autre part, empêchent de discriminer une phase de délibération et une phase de décision ${ }^{10}$.

En conséquence, la décision - toujours entendue comme l'arrêt d'une intention d'entreprendre une action - semble émerger directement de la discussion. Ce trait est particulièrement pertinent pour caractériser de manière descriptive une formulation de l'idéal de la discussion rationnelle. Je pense ici à certaines des réflexions sur la démocratie délibérative, celles qui promeuvent un modèle de la décision collective selon lequel la discussion, limitée à un échange d'arguments entre partenaires égaux, doit aboutir à une décision par le moyen d'un consensus (Cohen [1989]). Ce dernier est assuré par l'hypothèse et le souhait que chaque participant soit guidé dans la détermination de sa volonté par la force du meilleur argument (Chambers [1996]). La manière de constater ce consensus n'est pas mentionnée, un vote avec requête d'unanimité n'est pas évoqué. Habermas (1997) envisage bien le recours au vote à la majorité dans le cas où une décision devrait être prise sans qu'un consensus ait été atteint; il admet donc la possibilité d'une séparation entre la délibération et l'arrêt de la décision, quand le consensus s'avère impossible, mais cela laisse pendante la question de savoir comment un tel consensus, lorsqu'il est réalisable, est constaté. La formulation de cet idéal de la discussion rationnelle et de ce que doit être la délibération, s'attache à lister les exigences qui devraient être satisfaites. Mais ces auteurs, comme il est courant dans le cadre d'un travail de proposition normative, ne se plient pas à l'exigence descriptive, évoquée plus haut, qu'un observateur devrait satisfaire pour rendre compte d'une discussion rationnelle ou d'une délibération, au cas où les modèles idéaux de ces réflexions sur la démocratie délibérative pourraient être réalisés. Or, si l'on se plie à cette exigence descriptive, à savoir montrer comment les participants se rendent compte qu'il y a arrêt, force est d'admettre que, faute d'un vote à l'unanimité (fût-ce par hochement de tête), le modèle descriptif de l'aboutissement de la discussion rationnelle ou de la délibération (telle qu'elle est imaginée par une partie du courant «démocratie délibérative») est la décision par consensus apparent.

\subsection{La préférence pour un consensus de qualité}

La décision par consensus apparent est utilisée dans des contextes de décision collective où l'on attache de l'importance à une certaine conception du consensus. Cette conception associe étroitement approbation et qualité de la décision. Le souci d'un «consensus de qualité » est observable aussi bien dans la palabre que dans les aréopages.

${ }^{10}$ Cette continuité entre discussion et décision explique en partie le fait que certaines idées développées dans cette deuxième partie rencontrent celles, très éclairantes, de Pasquino (2006) qui part d'une distinction heuristique, voter et délibérer, légèrement différente de la mienne qui porte sur le mode d'arrêt de la décision. 
Emmanuel Terray souligne que la pratique de la palabre va de pair avec une certaine conception de la décision selon laquelle «à tout problème correspond une solution juste et une seule; l'objet de la discussion est alors de la trouver: non pas de l'inventer, mais de la découvrir. Les individus n'ont sur la réalité qu'un point de vue partiel: c'est donc la confrontation des opinions qui conduit progressivement au dévoilement de la vérité » (Terray [1987-89], p. 21). Les fondateurs de la commission française d'AMM avaient à peu près le même discours: «Personne ne possède la vérité, il faut écouter tous les points de vue, la bonne décision résulte de leur mise en commun et de la discussion ».

De tels propos manifestent clairement les mérites attribués à la confrontation des points de vue. Mais il est remarquable que, dans le cas de la palabre comme dans celui de la commission d'AMM, la valorisation de la délibération aille de pair avec le rejet du vote. Ce rejet ne va pas de soi, car, après tout, on peut voter après que les opinions ont été enrichies par la discussion ${ }^{11}$. Le consensus apparent s'oppose ici une nouvelle fois au vote par deux aspects étroitement articulés :

A. la recherche conjointe de la qualité de la décision et du nombre de ceux qui l'approuvent;

B. le consensus n'est pas une sommation d'opinions individuelles.

A. Là où la décision par consensus apparent est la manière légitime de prendre des décisions, les participants ne renoncent pas à joindre le nombre et la sagesse, à rebours de ce que nous montre l'émergence historique des techniques du vote et l'histoire de la réflexion sur celles-ci. Les Grecs soulignaient déjà la possibilité d'un écart entre les choix du peuple, numériquement supérieur, et les voies de la sagesse politique (Thucydide, VI, 24). Les ordres monastiques d'Occident qui ont réinventé les techniques électorales ont mis plusieurs siècles à admettre que le nombre (la majorité) puisse peser davantage qu'une minorité supposée éclairée dans la désignation du responsable des monastères: il leur fallut six cents ans pour laisser la major pars dominer sans pondération la sanior pars (Moulin [1958]). L'histoire de la réflexion sur les choix collectifs présente un mouvement similaire: l'évolution est allée de l'espérance de la découverte d'une bonne règle d'agrégation pour prendre une bonne décision (c'était l'espoir de Condorcet qui parlait de «décision vraie») à la recherche moins ambitieuse des règles dont le résultat «représente » la distribution des préférences individuelles de la moins mauvaise manière possible (Black [1958]; Arrow [1951]; Guilbaud [1952]). La recherche de la représentativité a remplacé le souci de la qualité. La disjonction générée par le vote entre la recherche de la qualité de la décision supposée diriger la discussion et le constat de l'addition des voix est irréductible. Elle reste présente et est même encore plus saillante quand on cherche à en diminuer l'ampleur. C'est le cas du vote motivé: pour limiter l'influence d'intérêts illégitimes, il arrive qu'on exige des membres d'un groupe délibérant que leur vote soit public et

${ }^{11}$ Les assemblées devant arrêter une décision collective peuvent présenter les trois cas de figures suivants: 1) passage direct au vote, une autre assemblée ayant la charge de la délibération et de la fixation des options en concurrence (Pasquino [2006]); 2) délibération puis vote; 3) délibération et absence de vote, la décision étant arrêtée par consensus apparent. 
motivé. Chacun doit justifier son vote par une argumentation acceptable. Le procédé a une limite bien connue: l'argumentation n'empêche pas la duplicité puisqu'un bon orateur trouve aisément des raisons acceptables pour justifier un vote qui a des motifs moins avouables ${ }^{12}$. Dans la décision par consensus apparent, la recherche du consensus n'est pas dissociée du souci de la qualité de la décision ${ }^{13}$. Dans les contextes où elle prévaut, le recours au vote est rejeté parce qu'il risque de sacrifier la qualité de la décision. Comme le disait l'un des experts de la commission d'AMM: «Dans la majorité des cas, il n'y a pas de vote. Ça peut étonner. C'est fondamentalement très bien. Ça veut être une décision scientifique. Donc, ça doit être un consensus. Sinon c'est qu'il reste des choses mystérieuses.» Via le consensus apparent, le nombre et la sagesse doivent se confondre et non s'opposer. Le vocabulaire de philosophie politique moderne congruent avec l'esprit de cette règle d'arrêt est celui du Contrat social de Rousseau: on attend de la volonté de tous (le nombre) qu'elle « déclare» la volonté générale (la qualité).

B. Si le consensus suppose bien l'approbation du plus grand nombre, par défaut de contestation, il ne se confond pas avec la sommation des opinions. Les ethnologues soulignent ce point quand ils expliquent que la palabre s'exerce dans un contexte normatif d'où l'individualisme politique est absent (Terray [198789]; Abélès [2003]). On comprend que l'absence de technique de sommation des opinions va de pair avec une conception de la légitimité des décisions sans rapport avec l'idée d'une sommation des citoyens-souverains, mais leurs descriptions ne permettent pas de saisir à quoi cela correspond exactement dans le processus même de la discussion. Les descriptions des aréopages contemporains sont sur ce point plus précises. Pasquino, songeant à la cour constitutionnelle italienne, explique qu'un juge ne saurait faire valoir «son opinion» auprès de ses partenaires, comme une volonté méritant, en tant que telle, de peser à titre de «fragment égal » de la souveraineté. Il ne peut espérer peser au nom de sa préférence pour l'option $\mathrm{x}$; seule vaut sa proposition d'une «bonne raison de choisir $\mathrm{x} \gg$. L'argument remplace la voix; la confrontation et finalement l'intégration des arguments remplacent l'agrégation des préférences (Pasquino, 2006). De la même manière, un expert de la commission d'AMM ne doit pas espérer peser sur la décision du seul fait qu'il ait exprimé son opinion pour ou contre l'autorisation d'un médicament ou pour ou contre telle recommandation associée à son autorisation. Son opinion ne vaut que parce que son argument a convaincu, est repris par les autres ou n'est pas contesté ${ }^{14}$.

${ }^{12}$ Les disputes au sein de l'assemblée des ingénieurs des Ponts et Chaussées qui, sous le Consulat, substitua le vote au consensus, en fournissent une très belle illustration (Graber [2004, 2005]).

${ }^{13}$ Quand il y a vote, le souhait de parvenir à une bonne décision est aussi présent, mais il n'est pas intégré d'une manière ou d'une autre au mécanisme de l'arrêt. Chacun peut espérer que la discussion ait été féconde, mais une fois que la procédure électorale est lancée, il n’y a plus qu'à constater le résultat agrégé.

14 Je dois cependant admettre, comme me le fait remarquer Bernard Manin, que l'exemple des aréopages tend à associer trop étroitement l'absence de contestation, requise par le consensus apparent, à l'exigence argumentative. Or il est possible de concevoir, à l'instar d'El-hakim, la décision par consensus apparent comme un mécanisme d'expression et d'agrégation des préférences sans argumentation. 
Ainsi la décision par consensus apparent présente-t-elle un contraste paradoxal avec le vote et notamment le vote à l'unanimité: elle est moins exigeante quant à la manifestation de la convergence des opinions puisqu'une apparence de consensus suffit; mais l'attente associée à ce consensus est plus forte, puisqu'il doit attester la qualité de ce qui va être décidé.

\subsection{Qui ne dit mot consent!}

La décision par consensus apparent est possible parce que certains consentent à ne pas s'opposer à la proposition de consensus. Nombre d'auteurs ont souligné que la palabre n'exige pas une unanimité fermement établie et que, le plus souvent, une minorité se rallie à l'option qui semble faire consensus, les opposants finissant par renoncer à contester (par ex. Dumont [1967], p. 227; Silberbauer [1982], pp. 32-34). Dans les aréopages, tous les membres n'approuvent pas complètement les décisions qui sont finalement prises, en partie grâce à leur silence. De la même manière, nous avons vu que dans les cas rassemblés sous le nom d' «expédients», des opposants à la proposition avancée ne cherchaient pas à la contester et ne demandaient pas un vote comme ils avaient la possibilité de le faire.

Dans le cas d'un vote à la majorité, simple, absolue ou qualifiée, une forme de consentement est également présente. Mais elle est inscrite dans l'adhésion au principe de la règle de majorité et dans son bon fonctionnement: la minorité accepte que la décision suive l'opinion de la majorité. Suivre la règle de majorité expose automatiquement à un tel consentement. Dans le cas de la décision par consensus apparent, le consentement est plus actif et plus personnalisé parce qu'il concerne directement l'expression des participants: dès lors qu'apparaît, par tâtonnement et par une succession de propositions, une tendance rassemblant de moins en moins d'opposants explicites, les derniers d'entre eux doivent se demander s'il vont ou non renoncer à exprimer leur désaccord. Pourquoi renoncent-ils ? Puisque la décision par consensus apparent semble accorder un droit de veto à chaque participant, à l'instar du vote à l'unanimité, la question des motifs et des raisons de ce renoncement mérite en effet d'être posée. Cette question rencontre dans la littérature deux types de réponses.

La première a trait aux intérêts des acteurs et aux rapports de force qui les lient. Les opposants se taisent parce que tel est leur intérêt au regard de la capacité de rétorsion que détiennent à leur encontre les principaux partisans de la proposition avancée. El-Hakim souligne que les notables savent exprimer, par des gestes ou des intonations de la voix, l'importance qu'ils attachent ou non à une décision et que, parallèlement, celui qui s'oppose à un consensus proposé ou soutenu par tel notable ayant la main sur la répartition individuelle de biens collectifs (par ex. le responsable de la distribution d'eau ou celui du dispensaire) peut craindre d'en subir la mauvaise volonté quand il aura besoin de ces biens. De la même manière, nous avons mentionné que les membres du conseil d'administration du FRAC n'osaient pas s'opposer au coup de force de leur président, déguisé en proposition de consensus, parce que ce dernier était une figure montante de la région et de leur parti politique.

La seconde réponse insiste sur la dimension normative: à un moment donné, la contestation est illégitime et suscitera la réprobation. Ainsi, explique Terray, 
dans la mesure ou la palabre est conçue comme une sorte de quête de vérité ou de bonne solution à un problème, «à partir du moment où celle-ci surgit, le débat s'arrête; tout propos supplémentaire serait superflu. La solution juste, c'est celle qui est la plus favorable au bonheur de la communauté: elle doit donc rallier l'unanimité des suffrages; dès qu'elle a été énoncée, celui qui continuerait à s'y opposer révélerait par là même qu'il préfère son intérêt particulier à l'intérêt général» (Terray [1987-89], p. 21). Encore une fois, la description de l'ethnologue ne permet pas de satisfaire notre curiosité. On aimerait savoir comment les participants peuvent avoir le sentiment que la «solution juste» a été proposée, ce malgré la persistance de désaccords. A partir de quels indices peuvent-ils estimer que les objections jusque-là légitimes sont désormais mal venues? Là encore, les observations plus aisées des aréopages contemporains mettent sur la voie. Imaginons un expert de la commission d'AMM qui a eu l'occasion de faire des objections à une proposition ou à un aspect de la proposition du président de séance sur le sort à réserver à un médicament. Ses objections ont déjà fait l'objet de la réflexion collective et ont été, par exemple, en partie intégrées dans la décision et en partie rejetées, ce avec des arguments qui semblent avoir convaincu les autres participants. Si ce même expert continue à s'opposer à la proposition reformulée du président de séance, avec des arguments semblables à ceux déjà réfutés, il suscite l'étonnement et la réprobation. Son engagement dans cette décision paraît excessif et éventuellement soupçonnable. Son entêtement est déplacé parce qu'il se comporte comme si son opinion avait une valeur en tant que telle, alors que sa valeur est indexée sur le succès de ses arguments. Il en irait de même si un juge de la cour constitutionnelle italienne, évoquée par Pasquino (2006), avait une attitude semblable. Ici, le consentement a pour levier l'épuisement des objections acceptables. Il se peut que, après un long débat et plusieurs objections, la dernière proposition de consensus ne m'agrée toujours pas, pour quelques raisons ou motifs; mais je n'ai plus la capacité de m'y opposer par des arguments estimés valables au regard du contexte normatif et de la rhétorique à l'œuvre dans l'assemblée.

Comment l'explication du consentement doit-elle pondérer la force des intérêts et l'autorité des arguments? El-Hakim (1978) ne prend en compte que les intérêts, faisant de la décision par consensus une forme complexe d'échange social, entendue au sens de Coleman (1966), dont la dynamique est assurée par les variations de la distribution des ressources et des capacités de contrôle des différents participants sur les autres. Cette option nous paraît trop exclusive, bien que, faute de description détaillée, on ne puisse trancher dans un sens ou dans un autre. Toutefois, dans la palabre, les décisions en cause touchent souvent les intérêts directs et personnels des participants, aussi l'hypothèse la plus plausible estelle que s'exercent en faveur du consentement aussi bien les intérêts que les normes collectives, sans que l'on puisse se représenter leur articulation. En revanche, dans les aréopages contemporains, les sages ou les experts n'ont généralement pas d'intérêts directs en jeu. Aussi peut-on s'attendre à ce que, même s'ils peuvent avoir des convictions fortes et s'engager vivement dans le débat, les normes de l'argumentation régissent quasi exclusivement les conditions du consentement.

Conformément à l'adage «Qui ne dit mot consent», l'arrêt de la décision par consensus apparent suppose que ceux qui n'approuvent pas la proposition ou qui n'y adhèrent pas complètement consentent à ne plus s'exprimer. 


\title{
2.6. Egalité de participation, inégalité légitime des influences
}

Les partisans comme les observateurs de la décision par consensus soulignent qu'elle s'appuie sur un principe d'égale participation de tous les membres. Par ailleurs l'exigence et le temps de parvenir à un consensus encouragent et permettent cette participation. C'est l'un des points forts de la valorisation de la palabre, souvent mis en avant par ceux qui veulent en souligner le caractère démocratique (Bidima [1997]; Mandela [1995]; Martin [1998]; Sen [2005]).

Mais l'égalité quant à la participation ne doit pas occulter l'inégalité, de fait et de droit, du poids des différents individus dans la formation du consensus apparent. Alors que le vote, notamment à la majorité, assure que, de fait, chaque électeur pèse exactement le même poids dans le résultat final - un homme, une voix la délibération et la décision par consensus apparent ne peuvent garantir et ne prétendent pas assurer l'égalité des contributions à la décision.

Pasquale Pasquino note à juste titre que l'égalité de principe des membres d'une assemblée délibérante se heurte à des différences factuelles, sources d'inégalités d'influence ${ }^{15}$. J'ajouterai, pour la décision par consensus apparent, que non seulement il y a des différences de fait qui se traduisent par des inégalités de poids dans la décision, mais que, de plus, ces inégalités sont jugées légitimes. Elles motivent d'ailleurs le rejet de la procédure du vote. Terray a eu l'occasion de discuter des mérites respectifs du vote et de la palabre avec les membres de la société Abron qu'il a étudiée. L'égale influence de chaque électeur sur la décision leur semble injustifiée: "l'idée que tous les suffrages pèsent d'un poids égal leur paraît tout à fait saugrenue: il y a des hommes intelligents et il y a des imbéciles, il y a des vieillards expérimentés et il y a d'innocents blancs becs » (Terray [198789]). Il en va de même dans la commission française d'autorisation de mise sur le marché des médicaments. Selon les experts de cette commission, les différences de compétences entre les membres, dont la pertinence varie constamment avec les différentes questions traitées, doivent avoir leur traduction dans le degré d'influence de chacun sur la décision finale. Les deux extraits d'interviews suivants le manifestent clairement:

\begin{abstract}
«Il ne faut pas voter, car cela donne le même poids à chacun. Si on examine le dossier d'un médicament pour le cœur, mon collègue cardiologue doit avoir plus de poids que moi.»

«On arrive toujours à un consensus, c'est bien mieux. Il n'y a jamais eu de vote. Vaut mieux que les gens s'expliquent. Que ceux qui sont compétents s'expliquent. Il y a des membres de droit qui représentent les académies de médecine, de pharmacie, l'Inserm. Ils sont représentatifs mais ils n'ont pas une compétence. Les experts sont compétents. Donc le problème du vote c'est qu'il donne un même poids à tous.»
\end{abstract}

Ainsi l'usage de la décision par consensus apparent est-il congruent avec la légitimité des inégalités des contributions individuelles à la décision collective. Le souci de l'égalité en matière de participation y côtoie la reconnaissance des inégalités d'influence.

${ }^{15}$ Il en note cinq: l'autorité personnelle, la compétence technique, la puissance argumentative, l'ordre des prises de parole, le rôle de président de séance. 


\section{CONCLUSION}

Mon objectif était de cerner la spécificité d'un mode de décision collective habituellement dénommé « décision par consensus » ou « décision à l'unanimité». Dans la première partie, il est apparu que c'était la description précise de la technique d'arrêt de la décision qui permettait de distinguer clairement ce que j'ai finalement appelé la «décision par consensus apparent» du vote à la majorité ou à l'unanimité. Cette distinction a autorisé un premier inventaire sommaire des usages de cette règle de décision. Dans la deuxième partie, j'ai examiné six propriétés de la décision par consensus apparent qui, par contraste avec le vote, ont notamment mis en évidence certains aspects normatifs qui accompagnent, dans la palabre et les aréopages, la pratique de cette manière d'arrêter la décision. Pour conclure, j'aimerais mentionner trois leçons.

1) Le contexte normatif de la décision par consensus apparent. Les aspects normatifs mis en évidence dans la deuxième partie invitent à distinguer nettement l'usage du consensus comme expédient, listé dans la première partie, de son usage dans les aréopages et la palabre. Quand il est utilisé comme un expédient, l'arrêt par consensus apparent est moins légitime que le vote avec lequel il coexiste et n'est donc jamais très loin d'une forme dégradée de vote informel. Ce type mixte et instable tend à montrer qu'une forme d'arrêt de la décision est congruente avec des idées et des normes attachées à la décision et à son contexte.

2) Décision par consensus apparent et démocratie délibérative. Les promoteurs d'une démocratie plus délibérative ont raison d'évoquer les exemples de pratiques telles que la palabre. Toutefois, ils n'en retiennent qu'un aspect, l'égalité de la participation aux débats, en négligeant un autre pourtant indissociable: l'inégalité des participants quant au poids de leur contribution au résultat final. Seul le vote assure que chaque électeur a la même influence que tous les autres. Si l'autorité du meilleur argument doit l'emporter dans la décision et si la délibération ne débouche pas sur un vote, la décision par consensus apparent, qui est le modèle descriptif de la décision par discussion rationnelle, suppose légitime et génère de fait l'inégalité des participants face à la décision collective. Il apparaît ainsi que le souci d'une participation plus égalitaire à la délibération heurte frontalement la conception de l'égalité politique qui a jusqu'ici prévalu dans les démocraties occidentales et qui a présidé à l'émergence du suffrage universel ${ }^{16}$. Il est d'ailleurs remarquable que la « démocratie des autres» que Sen (2005) détecte dans les traditions non occidentales, qualifiée de «vision beaucoup plus large de la démocratie en terme de débat public » (p. 15), soit dépourvue de passion égalitaire.

3) Deux distinctions utiles pour la comparaison. Il me semble que la prise en compte de la spécificité de la décision par consensus apparent enrichit les bases

16 «L'égalité politique rapproche et annule ce qu'il y a de plus naturellement différent entre les hommes: le savoir et le pouvoir. C'est la forme d'égalité qui est la plus artificielle et la plus exemplaire à la fois... Le suffrage universel... est indissociablement signe et réalité, chemin montré du doigt et égalité déjà là », Rosanvallon, 1992, pp. 15-16. 
conceptuelles de l'analyse comparative des décisions collectives dans les différentes sociétés humaines (dont Detienne [2003] offre un bel exemple). Quand ils sont correctement distingués, il apparaît que le consensus apparent et le vote sont congruents à des contextes normatifs différents. Pour autant, leur distribution ne respecte pas exactement une partition entre les sociétés modernes et les autres. Bien qu'en l'état de la littérature, il soit difficile de ne pas associer la diffusion des techniques électorales à l'histoire de l'Occident, il serait faux de réserver le vote aux sociétés modernes et la décision par consensus apparent aux sociétés non modernes. En effet, d'une part, l'usage du consensus apparent est la norme de décision dans certains aréopages, organes délibératifs non élus observables dans les sociétés modernes. Et, d'autre part, si la prédominance du consensus apparent est avérée dans nombre de sociétés non modernes, rien ne permet d'écarter l'occurrence dans ces sociétés de formes de vote, éventuellement sans technique électorale. La distinction entre, d'un côté, la décision par consensus apparent et, d'un autre côté, un arrêt de la décision par un dénombrement des opinions, me paraît donc centrale pour la réflexion comparative.

Elle doit être associée à une distinction logiquement antérieure que j'ai mentionnée rapidement (introduction de 1.) entre la présence de décision collective et son absence. Elle n'est qu'apparemment évidente: on risque toujours de projeter la notion de décision collective, qui nous est familière, sur des phénomènes d'assemblée où l'idée d'obligation, constitutive de la notion, est de fait absente. Ainsi certaines références à la règle d'unanimité peuvent nous égarer, en induisant l'idée d'une décision collective, là où seuls ceux qui approuvent une option sont censés l'entreprendre (Simmel [1999]).

Mais l'objet de cet article était la première distinction. Ainsi avons-nous montré que la décision par consensus apparent n'était pas une forme dégradée ou informelle du vote à l'unanimité. D'une part, parce que la différence pertinente ne tient pas à l'usage ou non d'une technique aisément repérable. D'autre part, parce que les recours à la majorité ou à l'unanimité discriminent deux espèces d'un même genre: le vote. La distinction qui permet d'isoler la décision par consensus apparent, et de l'opposer clairement au vote, porte sur un aspect plus essentiel de la décision collective: la modalité de son arrêt. Il s'agit de la distinction entre, d'un côté, le dénombrement des avis préalablement exprimés par les participants et, de l'autre côté, une proposition de consensus qui n'est plus contestée. La prégnance du modèle du vote dans nos sociétés tend à nous empêcher de saisir la nature spécifique d'une autre manière d'arrêter les décisions collectives: à la fois positive, faire une proposition, et négative, constater l'absence d'objections.

Centre de Sociologie du Travail et des Arts, CNRS, et EHESS, Paris

\section{BIBLIOGRAPHIE}

Abélès, Marc, Les lieux du politique, Société d'ethnographie, 1983.

Abélès, Marc, «Revenir chez les Ocholos », in Detienne, Marcel (ed.), 2003, pp. 393-413.

Alès, Catherine, Entre cris et chuchotements. Organisation sociale et symbolique des Yanomami. Paris, Editions Karthala, 2006. 
Arrow, Kenneth, Choix collectif et préférences individuelles, Calmann-Lévy, 1974 (1951).

Baechler, Jean, Démocraties, Calmann-Lévy, 1994.

Bailey, Fredrick George, 1965, «Decisions by consensus in councils and committees», in Banton M.(ed.), Political systems and distribution of power, Tavistosck, London.

Barnes, John., «Class and Committees in a Norwegian Island Parish », in Human Relations, VII, 1954, pp. 39-58.

Bidima, Jean-Godefroy, La palabre. Une juridiction de la parole, Michalon, 1997.

Black, Duncan, The Theory of Committees and Elections, Cambridge University Press, 1958.

Byock, Jesse, Viking Age Iceland, Penguin Books, 2001.

Chambers, Simone, Reasonable Democracy. Jürgen Habermas and the Politics of Discourse, Cornell University Press, 1996.

Cohen, Joshua, «Deliberation and Democratic Legitimacy», in Hamlin, Alan and Petit Phillip, eds, The Good Polity, Blackwell, 1989.

Coleman, James, «Foundations for a theory of collective decisions », American Journal of Sociology, $1966, \mathrm{n}^{\circ} 71$, pp. 615-627.

Detienne, Marcel (ed.), Qui veut prendre la parole?, Le genre Humain, n 40-41, Seuil, 2003.

Dumont, Louis, Homo Hierachicus, Gallimard, Tel, 1966.

Dumont, Louis, Essais sur l'individualisme, Seuil, 1983.

El-Hakim, Sherif, «The Structure and Dynamics of Consensus Decision-Making», Man, vol. XIII, 1978.

Ferme, Mariane, «The Violence of Numbers: Consensus, Competition and the Negociation of Diputes in Sierra Leone», Cahiers d'Etudes Africaines, 150-152, vol. XXXVIII, 1998, pp. 555-580.

Ferejohn, John, Pasquino, Pasquale, «Consititutional Courts as Deliberative Institutions », in Alavarez, Sadurski (ed.), Consitutional Justice, East and West, Kluwer Law International, 2002.

Fishkin, James, Democracy and Deliberation, Yale University Press, New Haven, 1991.

Gaudemet, Jean, Les élections dans l' Eglise latine, Fernand Lanore, 1979.

Gilbert, Margaret, Marcher ensemble. Essais sur les fondements des phénomènes collectifs, PUF, collection Philosopher en sciences sociales, 2003.

Goscinny, René, Uderzo, Albert, Astérix. Le glaive et la rose, Les éditions Albert René, 1991.

Graber, Frédéric, La délibération technique. Disputes d'ingénieurs des Ponts et Chaussées sous la Consulat, Thèse de doctorat EHESS, 2004.

Graber, Frédéric, «Obvious decisions. Decision-making among French Ponts-et-Chaussées engineers around $1800 »$, Working paper, 2005.

Guilbaud Georges-Théodule, «Les théories de l'intérêt général et le problème de la logique de l'agrégation », Economie appliquée, 15, 1952, pp. 509-511.

Habermas, Jürgen, Droit et démocratie. Entre faits et normes, Gallimard, 1997.

Hauray, Boris, Contrôler les médicaments dans un espace européen. Politique, expertise et influence des firmes, Presse de Sciences-Po, 2005.

De Jouvenel, Bertrand, De la politique pure, Calmann-Lévy, 1977.

Moulin, Léo, «Sanior et major pars. Note sur l'évolution des techniques électorales dans les ordres religieux du VIe au XIIIe siècle», Revue Historique de Droit Français et Étranger, 1958, vol. XXXV.

Mahé, Alain, «Les assemblées villageoise dans la Kabylie contemporaine », Etudes Rurales, $\mathrm{n}^{\circ} 155$ 156, juillet-décembre 2000, pp. 179-212.

Mandela, Neslon, Un long chemin vers la liberté, Fayard, 1995.

Martin, Pierre, «Réconcilier délibération et égalité politique: Fishkin et le sondage délibératif», Revue Française de Science Politique, ${ }^{\circ}{ }^{1}$, vol. 48, février 1998, pp. 150-154.

Pasquino, Pasquale (2007), «Voter et délibérer», Revue Européenne des Sciences Sociales, à paraître. 
Popkin, Samuel, The Rational Peasant. The Political Economy of Rural Society in Vietnam, University of California Press, London, 1979.

Richards, Audrey and Kuper, Adam (ed.), Councils in Action, Cambridge papers in Social Anthropology, Cambridge University Press, 1971.

Raz, Joseph, «Reasons for Action, Decisions and Norms », Mind, vol. 84, n 336, oct. 1975, pp. 481499.

Rosanvallon, Pierre, Le sacre du citoyen, Folio Gallimard, 2001.

Sen, Amartya, La démocratie des autres, Editions Payot et Rivages, 2005.

Silberbauer, George, «Political Process in G/wi bands », in Leacock Eleanor and Lee Richard, Politics and history in band Societies, Cambridge University Press/ed. De la Maison des sciences de l'homme, New York/Paris, 1982, pp. 23-36.

Simmel, Georg, Sociologie, PUF, 1999.

Smith, Thomas, The agrarian Origins of Modern Japan, Stanford University Press, Stanford, 1959.

Souyri, Pierre François, «Des communautés monastiques dans le Japon médiéval », in Detienne, Marcel, 2003, pp. 85-94.

Steiner, Jürg, and Dorff, Robert, «Decision by Interpretation: A New Concept for an Often Overlooked Decision Mode», British Journal of Political Science, vol. 10, 1980, pp. 1-13

Terray, Emmanuel, «Un anthropolgue africaniste devant la cité grecque», Opus. Rivista internazionale per la storia economica e sociale dell' antichità, VI-VIII, 1987-1989.

Terray, Emmanuel, «Le débat politique dans les royaumes de l'Afrique de l'Ouest. Enjeux et formes », Revue Française de Sciences Politiques, vol. 38, n 5, Octobre 1988.

Thucydide, La guerre du Péloponnèse, Gallimard, Folio, 2000.

Turton, David, «The Relationship Between Oratory and Exercice of Influence Among the Mursi », in Bloch Maurice, Political Language and Oratory in Traditional Society, Academic Press, London, 1975, pp. 163-184.

Urfalino, Philippe, La décision fut-elle jamais un objet sociologique?, document de travail, 2005, disponible sur le site CESTA-EHESS.

Urfalino, Philippe (2006), «La communauté des experts décideurs», soumis à Annales HSS.

Urfalino, Philippe, Vilkas Catherine, La délégation du jugement esthétique. Les Fonds Régionaux d'Art contemporain, L'Harmattan, 1995.

Vilkas, Catherine.,»Évaluations scientifiques et décisions collectives: le Comité national de la recherche scientique», Sociologie du travail, $\mathrm{n}^{\circ} 3,1996$, pp. 331-348.

Yngvesson, Barbara, «Leadership and Consensus: Decision-Making in an Egalitarian Community », Ethnos, n 1-2, 1978. 